\title{
The Method of Calculating the Spectral Sensitivity of Solar Cells
}

\section{Krutikov Vladimir Nikolaevich, Parfentiev Nikolay Andreevich}

All-Russian Research Institute for Optophysical Measurements (VNIIOFI), Moscow, Russia

\section{Email address:}

nik_parfenium@mail.ru (P. N. Andreevich)

\section{To cite this article:}

Krutikov Vladimir Nikolaevich, Parfentiev Nikolay Andreevich. The Method of Calculating the Spectral Sensitivity of Solar Cells. American Journal of Physics and Applications. Vol. 5, No. 6, 2017, pp. 106-108. doi: 10.11648/j.ajpa.20170506.16

Received: June 22, 2017; Accepted: July 5, 2017; Published: November 8, 2017

\begin{abstract}
A new method for calculation the spectral sensitivity of solar cells based on data obtained by measurement of differential sensitivity. As follows from the conducted analysis, measurements of cells can be carried out only at a constant spectral composition of background radiation. In particular with a single powerful lamp is repositionable relative to the cell. In the practical application of the method is expected to increase the accuracy of determining the spectral sensitivity, especially in the region of large wavelengths, where there is the maximum nonlinearity of the dependence of a current on irradiance.
\end{abstract}

Keywords: Spectral Sensitivity, Differential Sensitivity, Measurements of Solar Cells

\section{Introduction}

Among the alternative sources of energy the use of solar energy is the most environmentally friendly and promising. Metrology of solar cells allows to obtain the basic data for the design and operation of solar power plants [3-11]. The main difficulty in this process is caused by the nonlinearity of the current characteristics of solar elements (SE), spectral sensitivity of which depends on the full (total) irradiance solar cells. The present article is devoted to improvement of the specific method of measuring the spectral sensitivity of solar cells.

In work [1] is proposed the method of determining the two most important characteristics of solar cells: the short-circuit current of solar exposure - the spectral sensitivity under the same conditions. Since the solar cell is not a linear element (depend on level of radiation) measurement scheme is created in which the solar cell is irradiated simultaneously by two streams of radiation:

1 Spectral radiation - weak correctly recognized, modulated flux from the monochromator,

2 Background radiation - a powerful steady stream from the group of halogen lamps, whose number varies.

The result is an array of these differential sensitivity$S_{\text {diff }}(\lambda, I b)$, depending on the wavelength $-\lambda$ and the shortcircuit cell $-I b$. The true method of calculating short circuit current cell for standard conditions - $I_{S T C}$ is created in work [1]
The paper [1] contains also a description of the method of computation of the spectral sensitivity of the solar cells (SC) measured by their differential sensitivity to various levels of radiation. Formula for the calculation of spectral sensitivity $S_{S T C}(\lambda)$ at the selected wavelength $\lambda$ in standard irradiation conditions

$$
S_{S T C}(\lambda)=\frac{I_{S T C}}{\int_{0}^{I} I_{T C} \frac{d I}{S_{\text {diff }}(\lambda, I b)}}=\frac{1}{\frac{\int_{0}^{I} S T C \frac{1}{\overline{d i f f}_{(\lambda, I b)} d I}}{I_{S T C}}}
$$

$I_{S T C}$ - the calculated short-circuit current of the SC at a standard irradiance, $S_{\text {diff }}(\lambda, I b)-$ an array of the measurements of the differential sensitivity to the selected wavelength $\lambda$ depending on the current $\mathrm{SC}-I b$ arising from the source of illumination.

It follows from the last form (1), the spectral sensitivity is calculated as the reciprocal of the average (for bias current) reverse differential sensitivity. The proof of this formula has no physical basis.

In result, calculations by (1) lead to erroneous results, since small values of the differential sensitivity have more weight. Moreover, the spectral sensitivity becomes zero in the case when the magnitude of differential sensitivity on any level is set to zero (with any sensitivity at other levels). 


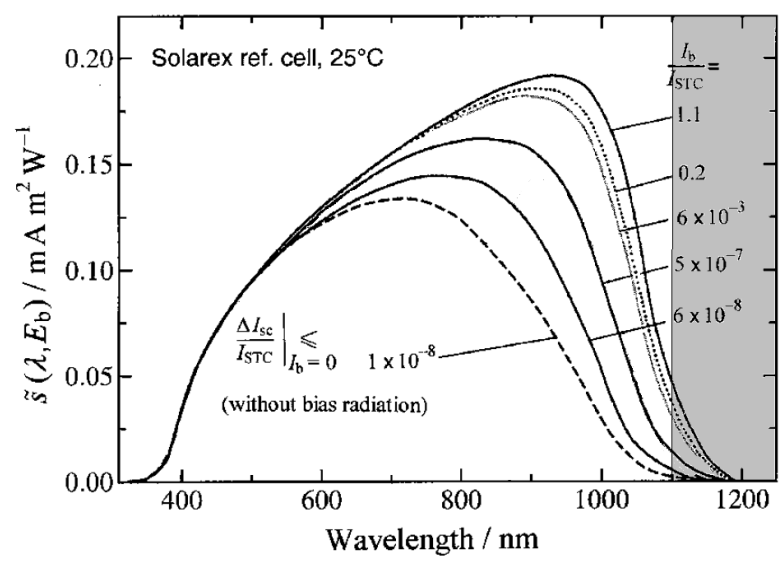

Figure 1. Spectral sensitivity of solar cell vs. wavelength and bias current.

In figure 1 (taken on [1]) the gray color indicates the area of the wavelengths above $1100 \mathrm{~nm}$. In this range of spectral sensitivity (and hence differential) is equal to a zero. It is easy to see that integration with respect to figure 1 will give a value of zero sensitivity.

It should be recognized that practical calculations of the sensitivity in the case, where they are held with limited range of currents cells (exclusion of small irradiation) not leads to substantial errors. However, the above formula involves integration from zero values of current and the calculation algorithm becomes uncertain. There is no reason for a reasonable choice of the lower limit of integration in form (1). Additionally, as shown above, process of measurements should maintain the constancy of the spectral composition of the background radiation.

In the present communication proposes a methodology of computation, free from the above errors.

\section{The Method of Calculation of Sensitivity}

The nonlinear sensitivity of the receiver at a particular wavelength can be found from its differential sensitivity by of normal integration, if known dependence of the differential sensitivity of the spectral magnitude of the irradiance. In particular, the sensitivity of the cell $-S(\lambda)$ at a certain wavelength $\lambda$ and maximal spectral irradiance $\operatorname{Em}(\lambda)$ can be represented as:

$$
S(\lambda)=\frac{\int_{0}^{E m(\lambda)} s_{\text {diff }}(\lambda, E(\lambda)) d E}{E m(\lambda)}
$$

$E(\lambda)$ and $E m(\lambda)$ - accordingly irradiance and maximum irradiance SC on wavelength $-\lambda$. Obviously, $E(\lambda)$ and $E m(\lambda)$ is spectral intensities of background radiation at this wavelength in the case of measuring differential sensitivity of solar cells.

Because of the differential sensitivity of the cell is derived as $f=\frac{d I}{d E}$, the integral in (2) represents the cell current is computed by differential sensitivity. The ratio of current to maximum spectral irradiance is the sensitivity of the cell to this wavelength of light conditions from the sun.
Normalizing irradiance by its maximum value, we get

$$
S(\lambda)=\int_{0}^{1} S_{\text {diff }}(\lambda, \alpha) d \alpha,
$$

where $\alpha$ is the ratio spectral radiances $E(\lambda) / E m(\lambda)$.

A further conclusion is made on the model proposed in [1] to determine the short-circuit current.

As applied to the measurement of the SC, energy irradiance SC - E(Ib) at the level of the current SC $I b$ can be calculated as

$$
E(I b)=\int_{0}^{I b} \frac{d I}{S_{s}(I b)}
$$

where $S_{S}(I b)$ sensitivity to standard illumination, calculated as

$$
S_{S}(I b)=\frac{\int_{0}^{\infty} S_{d i f f}(\lambda, I b) E_{S}(\lambda) d \lambda}{E_{S T C}}
$$

$E_{S}(\lambda)$ is the spectral distribution of the Sun, and $E_{S T C^{-}}$ solar constant.

Denoting the value of the integral at (5) as $I_{S}(I b)$ obtained

$$
E(I b)=E_{S T C} \int_{0}^{I b} \frac{d I}{I_{S}(I b)}
$$

Introduce the dimensionless coefficient $\alpha_{\mathrm{int}}$, is the ratio of the background irradiance of the SC, causing the current solar cell - $I b$, to the irradiance in standard conditions

$$
\alpha_{i n t}=\frac{E(I b)}{E_{S T C}}=\int_{0}^{I b} \frac{d I}{I_{S}(I b)}
$$

According to the formula (7) you can calculate the integral coefficient - the ratio of the integral of the background irradiance to irradiance, corresponding to solar radiation. In order to use this coefficient in (3) is necessary condition $\alpha=\alpha_{\text {int }}$ in all range of wavelengths, i.e. the spectral composition of the background radiation should be the same at all levels.

Coefficient $\alpha_{\text {int }}$ is values from zero to one when the irradiance, changing from 0 to the standard solar irradiance. Calculating $\alpha_{\text {int }}$ for all levels of current $I b$, we must convert two-dimensional array $S_{\text {diff }}(\lambda, I b)$ in an array of $S_{\text {diff }}\left(\lambda, \alpha_{i n t}\right)$, which depends on the wavelength and $\alpha_{i n t}$ the relative irradiance. Thus, the spectral sensitivity in standard conditions can be calculated by the formula (3).

According (3), large values of the differential sensitivity of yield correspondingly greater contribution to the value of the sensitivity in standard conditions. It is also evident that the lower limit of integration (3) can be assumed to be zero.

\section{A Modified Scheme for the Measurement of Differential Spectral Sensitivity of Solar Cell}

As shown above, measurements differential sensitivity can be done only with a constant spectral composition of background radiation. Background radiation levels may not 
vary from the number of included bulbs. Otherwise, all luminaries must be measured, showing the constancy of the spectral composition of all the used lamps. A more correct way of background radiation to use one powerful lamp, this is moved relative to the measured cell.

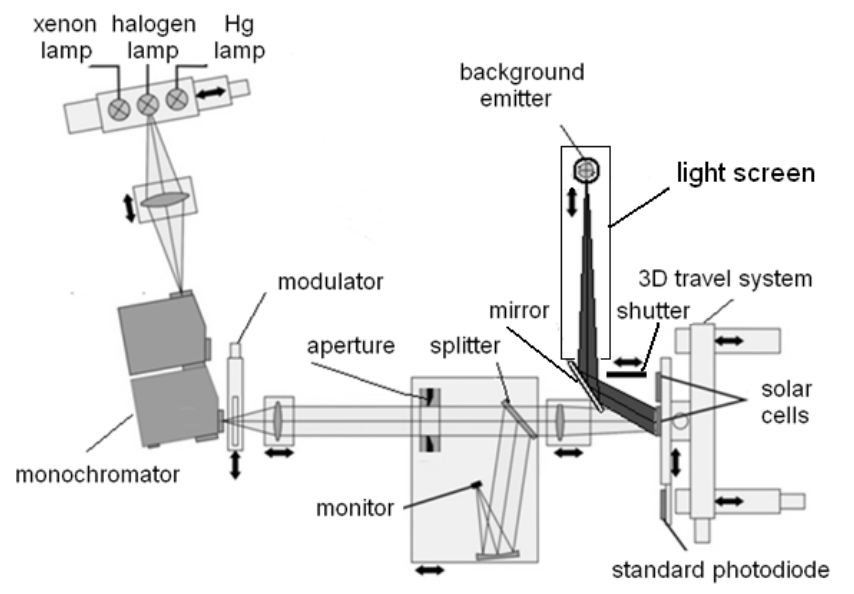

Figure 2. Diagram of the setup for measuring the differential sensitivity of solar cells.

In Figure 2 is a diagram of the modified setup to measure the differential sensitivity of solar cells. The fundamental difference of the proposed installation from the original prototype is the use of a single illuminator as a source of background radiation. The halogen lamp with power of $1 \mathrm{~kW}$ is used to simulate solar radiation. The lamp is moved relative to the cells at a distance from $150 \mathrm{~mm}$ to $1500 \mathrm{~mm}$, i.e. the level of background radiation varies 100 times. As follows from the prospective method, small values of the background irradiance does not give a significant contribution to the estimated sensitivity value. The spectral composition of the background radiation remains constant at all levels. The radiation of the lamps is overlapped by the shutter, which allows reducing duration of measurements excluded the time of setting the temperature regime of the cell. The lamp is moved in a light-proof casing to exclude the lateral flare of the solar cell.

Measurement scheme (similar to PTB) created in the Institute VNIIOFI with the original software [2]. Currently the scheme is being rebuilt, in order to carry out correct measurement of the differential spectral sensitivity. Measurement data will be processed as described above. Sorry, we have no data to calculate the sensitivity of cells in a new way. All measured cells were linear devices and to calculate their sensitivity is not required the algorithm. For this reason, we could not compare the previous and the new method of calculating sensitivity.

\section{Conclusion}

1. The method of determining spectral sensitivity is proposed on the base of data the differential sensitivity.

2. It is shown in case, when measuring the differential sensitivity, of the spectral composition of the background radiation must be constant.

3. The scheme of the modernized installation for the measurement of differential sensitivity of solar cells.

4. The use of the modified scheme of measurements allows reducing the total time of the measurement cells.

The developed method is proposed to use to improve the accuracy of measurements and calculations of the sensitivity of the SC under standard conditions. The method and setup is designed to standard measure the spectral sensitivity by of measurement of differential sensitivity.

\section{References}

[1] Metzdorf and all. Radiometry in voltaic: calibration of reference solar cells and evaluation of reference value. Metrologia, 2000, v. 37, p 573-578.

[2] Fidanyan, G. S., Morozova S. P., Parfentiev N. A. Setup for measuring the absolute spectral sensitivity of the solar cell under standard conditions. XXIV International scientifictechnical conference and school on Photonics and night vision devices. 22-27 May 2016, page 258-262. Moscow, Russia.

[3] W. Keogh and Blakers, A. W., "Natural Sunlight Calibration of Silicon Solar Cells", 17th European Photovoltaic Solar Energy Conference. Munich, Germany, 2001.

[4] K. Emery, Myers, D., and Rummel, S., "SOLAR SIMULATION - PROBLEMS AND SOLUTIONS", 20th IEEE PV Specialists Conference. p. 1087, 1988.

[5] D. King and Hansen, B., "A Sensitivity Analysis of the Spectral Mismatch Correction Procedure Using WavelengthDependent Error Sources", 22nd IEEE PV Specialists Conference. 1991.

[6] J. Zhao, A., W., Dai, X., Green, M. A., and Wenham, S. R., "Improvements in Silicon Solar Cell Performance", 22nd IEEE PV Specialists Conference. pp. 399-402, 1991. M. Wolf and Rauschenbach, H., "Series Resistance Effects on Solar Cell Measurements", Advanced Energy Conversion, vol. 3, 1963.

[7] R. A. Sinton and Cuevas, A., "A Quasi-Steady-State OpenCircuit Voltage Method for Solar Cell Characterization", in 16th European Photovoltaic Solar Energy Conference, Glasgow, Scotland, 2000, pp. 1152-1155.

[8] M. J. Kerr, Cuevas, A., and Sinton, R. A., "Generalized analysis of quasi-steady-state and transient decay open circuit voltage measurements", Journal of Applied Physics, vol. 91, p. 399, 2002.

[9] A. Mette and et al, "Series resistance characterization of industrial silicon solar cells with screen-printed contacts using hotmelt paste", Progress in Photovoltaics: Research and Applications, vol. 15, pp. 493-505, 2007.

[10] D. PYSCH, Mette, A., and GLUNZ, S., "A review and comparison of different methods to determine the series resistance of solar cells", Solar Energy Materials and Solar Cells, vol. 91, pp. 1698 - 1706, 2007.

[11] S. Bowden and Rohatgi, A., "Rapid and Accurate Determination of Series Resistance and Fill Factor Losses in Industrial Silicon Solar Cells", in 17th European Photovoltaic Solar Energy Conference, Munich, Germany, 2001. 